\title{
Opportunistic Multihopping for Energy Efficiency: Opportunistic Multi-Hop Cellular Networking for Energy-Efficient Provision of Mobile Delay Tolerant Services
}

\author{
Baldomero Coll-Perales, UWICORE Lab, Miguel Hernandez University of Elche \\ Javier Gozalvez, UWICORE Lab, Miguel Hernandez University of Elche \\ Oscar Lazaro, Innovalia Association \\ Miguel Sepulcre, UWICORE Lab, Miguel Hernandez University of Elche
}

Cellular operators are facing considerable capacity and energy-efficiency challenges as cellular data traffic keeps on rising. Several studies have shown that multi-hop cellular networks (MCN) could help tackle these challenges through the integration of device to device and cellular communications. This study demonstrates that significant energy-efficiency gains can be achieved when MCN networks jointly exploit opportunistic networking and the traffic delay tolerance characteristic of relevant mobile data services.

\section{Introduction}

Cellular data traffic is significantly growing with the pervasive use of smartphones and the popularity of mobile video, web browsing and social networking applications among others. This trend will do nothing but exponentially grow with machine-to-machine (M2M) applications [1]. An approach to address the capacity crunch is the dense deployment of small cells. However, provisioning "additional” infrastructure bears significant costs both at the deployment and the management phases. So, this solution in isolation might not be able to meet the data capacity demand for current and future networks. Other emerging alternatives currently being considered as part of $5 \mathrm{G}$ network evolution include the offloading of cellular data traffic to device to device (D2D) connections [2], and the integration of cellular, Wi-Fi and ad-hoc or D2D communications into what is referred to as multi-hop cellular networks (MCN) [3] $]^{1}$. The increasing processing power and networking capabilities of smartphones provide significant opportunities for the use of mobile relays in MCN (MCN-MR). In fact, a recent study experimentally demonstrated that MCNMR networks can increase quality of service (QoS) and network capacity, as well as reduce energy consumption [4]. Additional capacity and energy-efficiency gains could be further achieved through the integration of opportunistic networking [5] into MCN. These gains can be particularly

\footnotetext{
${ }^{1}$ EU projects such as MOTO (http://www.fp7-moto.eu/) and METIS (https://www.metis2020.com/) are studying these solutions.
}

relevant when taking advantage of the delay tolerance or nonreal-time properties of certain traffic services (e.g. email, file sharing, social networking, software/firmware updates, mobile video, cloud services, data metering, goods tracking); according to Cisco's global mobile data traffic forecast for 2013-2018², these services represent some of the most popular applications driving the mobile data growth. Delay tolerant services exhibit some relaxation in the time constraints for data transmission without impact on user quality of experience. This study exploits the data delivery margins offered by delay tolerant services to improve the energy-efficiency of cellular networks using opportunistic networking policies in MCN networks. To this aim, this study uses as a basis the optimization framework for 2-hop opportunistic MCN communications proposed by the authors in [6]. This optimization framework allows identifying energy-efficient configurations of end-to-end opportunistic MCN communications for delay tolerant services. To do so, the framework identifies the optimum location of the mobile relay and the location at which the relay should start forwarding the information to the cellular base station. The authors extended this first study to consider scenarios where the optimum configurations cannot be met, i.e. a mobile relay cannot be found when needed at the identified optimum location. In particular, the authors proposed in [7] to exploit context information to derive sub-optimum configurations that take into account the probability to find a mobile relay at the optimum location. [7] demonstrates that the identified suboptimum configurations can guarantee the end-to-end QoS requirements utilizing context information easily extracted from cellular networks. This study builds from previous results to report for the first time, the energy-efficiency gains that can be achieved with opportunistic MCN communications based on the traffic characteristics of delay tolerant services. In addition, this study investigates how opportunistic MCN

\footnotetext{
${ }^{2}$ Cisco Visual Networking Index: Global Media Data Traffic Forecast Update, 2013-2018, Cisco Whitepaper, Feb. 2014.
} 
communications should be configured to exploit traffic characteristics in order to reduce the energy consumption.

\section{Delay tolerant mobile traffic services}

Cisco estimates that mobile video represented 53\% of the mobile data traffic by the end of 2013. This percentage is expected to increase to $69 \%$ by 2018 . Even uplink mobile video traffic is increasing thanks to the popularity of applications such as Ustream, Bambuser or Livestream that allow users to broadcast live events using their mobile devices. Mobile video content delivery technologies currently utilize adaptive streaming (AS) schemes that divide video content into fragments. These fragments are delivered to the user on demand to ensure that sufficient content (in the order of tens of seconds) is constantly buffered at the user device ahead of playback time. This buffering policy avoids freezing of video streaming, thereby improving the user's quality of experience (QoE). The study in [8] showed that YouTube adaptive streaming delivers approximately 1 Megabyte (MB) of data to the user's player every 10 seconds, with the data being received in multiple fragments. However, video content fragments could also be grouped to postpone their transmission at no cost to the perceivable QoE as long as the playback is not interrupted [9].

Mobile email data traffic is another example of popular application that can tolerate certain transmission delays. In fact, email traffic does not require synchronous end-to-end communications and can tolerate delays (>>10s) according to the ITU QoS classification [10]. The delay tolerance is expected from the polling frequency at which email clients check for emails at servers. For example, Gmail clients check for emails approximately once per hour and the polling frequency reduces as long as emails are found on the server; the polling interval can be reduced to approximately 5 minutes intervals.

Social networks and messaging applications generate a significant amount of photo sharing and messaging (text and multimedia) traffic. Uploading photos to social networks is not necessarily an instantaneous process, and can be configured considering factors such as the synchronization frequency. Mobile messaging applications require a data connection at the transmitter and receiver for the exchange of messages. Mobile messaging applications are delay tolerant from their design since they postpone the transmission or reception of the messages if such connections are not available.

Other relevant delay tolerant applications include overthe-air (OTA) updating and cloud file hosting services. OTA updating services are becoming increasingly popular in smartphones and tablets for updating applications, software or firmware as well as configuration settings on mobile devices [11]. These services are delay tolerant in nature, and can be designed to exploit connectivity and offloading opportunities. Cloud file hosting services allow (mobile) users to store content in the cloud and access it at any time and place using different (mobile) devices/platforms. These services can be configured to automatically upload files of any type for backup or just for sharing with other users and platforms (e.g. photos or videos). This process can again be configured to exploit the characteristics and efficiency of connectivity conditions. The possibility to optimize the configuration of mobile uploading processes is also present in the vehicular environment. For example, car insurance companies are introducing pay-as-youdrive policies that calculate the drivers' insurance premiums based on the vehicle usage (speed, acceleration, time of day, type of road, and milestone). Vehicles are equipped with an accelerometer and a GPS to track the vehicle location/acceleration, and a cellular modem to collect the measurements at the control centre. The frequency with which the vehicle uploads the information is managed at the control centre [12]. Therefore, the upload of information from the vehicle can be deemed as delay tolerant.

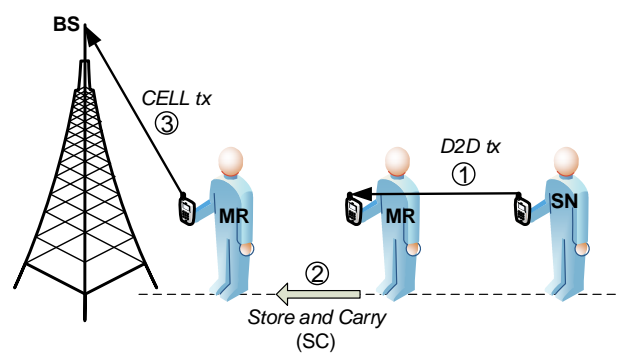

Figure 1 2-hop opportunistic MCN scenario.

\section{Energy consumption of opportunistic $\mathrm{MCN}$ networks with mobile relays}

This study considers the use of opportunistic networking in MCN networks to improve the energy-efficiency by exploiting the delay tolerance of relevant mobile data services. To this aim, the study focuses on the 2-hop uplink MCN scenario represented in Figure 1. In this case, the source node (SN) does not transmit its data directly to the base station (BS), but transmits it first to a mobile relay (MR) with improved link budget using D2D communications. The SN-MR and MR-BS transmissions can be real-time, or can be opportunistically scheduled for delay tolerant traffic services based on the quality and efficiency of the links. Opportunistic networking schemes can exploit such delay tolerance together with the nodes' mobility and the store, carry and forward paradigm to program the SN-MR and MR-BS transmissions so that the overall energy consumption for the end-to-end MCN radio transmission is minimized.

This study considers that a static SN wants to upload a message of size $F$ to the BS before a deadline $T$. The term message refers to the information data that needs to be uploaded to the BS before the service-dependent delay tolerance $(T)$ elapses. For example, it could represent the entire file in the case of cloud/email services, or a set of grouped 
fragments in the case of mobile video traffic. For the transmission of the message, SN can establish an opportunistic 2-hop MCN connection to the BS using MR as mobile relay (Figure 1). The time required to transmit the message needs then to consider the time needed to transmit the data from SN to MR $\left(t_{D 2 D}\right)$, the time the data is stored at the mobile MR $\left(t_{S C}\right)$, and the time needed by MR to upload the data to the BS $\left(t_{C E L L}\right)$. Finding an optimum opportunistic 2-hop MCN configuration that minimizes the energy consumption is then equivalent to determining the values of $t_{D 2 D}, t_{S C}$, and $t_{C E L L}$ that satisfy the following multi-objective constrained function:

$$
o . f: \min \left(E_{D 2 D}\left(t_{D 2 D}\right)+E_{S C}\left(t_{S C}\right)+E_{C E L L}\left(t_{C E L L}\right)\right)
$$

where $E_{D 2 D}, E_{S C}$ and $E_{C E L L}$ represent the energy consumed in the transmission from SN to MR, the store and carry process, and the transmission from MR to $\mathrm{BS}$, respectively. The objective function is defined together with the restrictions that the D2D and cellular data transmissions $(T R)$ of the message of size $F$ must be completed, and that the end-to-end transmission is completed before the service-dependent deadline $(T)$ :

$$
\begin{gathered}
T R_{D 2 D}(S N \rightarrow M R) \geq F \\
T R_{C E L L}(M R \rightarrow B S) \geq F \\
t_{D 2 D}+t_{S C}+t_{C E L L} \leq T
\end{gathered}
$$

The defined optimization problem can identify the optimum 2-hop opportunistic MCN configuration that minimizes the overall energy consumption for a given location of SN and certain traffic service characteristics ( $F$ and $T$ ). Finding such optimum configuration is equivalent to determining the MR location and the location at which the MR needs to start forwarding the information to the cellular network in order to minimize the energy consumption. Full details about the optimization problem can be found in [6].

\section{Energy models}

The energy consumption resulting from the cellular and D2D transmissions has been modelled taking into account that the transmission power $\left(P_{T}\right)$ is the necessary one to guarantee a power level at the receiver $\left(P_{R}\right)$ equal to a threshold needed for a successful transmission, and that the propagation losses follow the WINNER model for urban scenarios with low antenna heights. In this context, $P_{T}$ can be computed as a function of the distance $d$ (in meters) between the transmitter and the receiver for Line-Of-Sight (LOS) conditions [13]:

$$
P_{T}^{L O S}(d)= \begin{cases}\frac{P_{R} \cdot 10^{4.1} \cdot(f / 5)^{2}}{G_{T} \cdot G_{R}} d^{2.7} & \text { if } d<d_{b p} \\ \frac{P_{R} \cdot 10^{4.1} \cdot(f / 5)^{2}}{G_{T} \cdot G_{R} \cdot d_{b p}^{1.73}} d^{4} & \text { if } d \geq d_{b p}\end{cases}
$$

where $f$ represents the carrier frequency (in $\mathrm{GHz}$ ), $G_{T}$ and $G_{R}$ represent the transmitter and receiver antenna gain (here equal to 1 ), and $d_{b p}$ is the breakpoint distance. $d_{b p}$ is calculated as
$4 \cdot\left(h_{T}-1\right) \cdot\left(h_{R}-1\right) / \lambda$, where $h_{T}$ and $h_{R}$ represent the antenna heights of the transmitter and receiver (in meters), and $\lambda$ is the carrier wavelength (in meters). The energy consumed in the D2D $\left(E_{D 2 D}\right)$ and cellular $\left(E_{\text {cell }}\right)$ transmissions under LOS conditions can be estimated taking into account the energy consumption per bit in the electronics of the transmitter $\left(e_{t}\right)$ and receiver $\left(e_{r}\right)$ :

$$
E(d)=\left(e_{r}+e_{t}+e_{L O S}(d)\right) \cdot T R
$$

where $e_{L O S}$ represents the transmission energy consumption per bit under LOS conditions. $e_{L O S}$ is equal to $P_{T}{ }^{L O S} / T R$, where $T R$ represents the throughput $\left(T R_{D 2 D}\right.$ or $\left.T R_{\text {cell }}\right)$. The transmission energy consumption under Non-LOS (NLOS) conditions can be computed following a similar process.

This study considers, without loss of generality, that cellular transmissions utilize LTE at $2 \mathrm{GHz}$ and that D2D utilizes IEEE802.11g at $2.4 \mathrm{GHz}^{3}$. These radio access technologies were selected due to the availability of the necessary models for modelling cellular and D2D communications ([14] and [15] respectively). The conclusions obtained in this study could though be extrapolated to other radio access technologies. In fact, the authors have replicated the analysis considering HSPA for the cellular radio access, and the same conclusions have been observed.

Following [14], the cellular LTE throughput can be computed as a function of the distance ( $d$, in meters) between MR and BS:

$$
T R_{\text {cell }}(d)=r\left(N_{P R B}, l_{M C S}\right) \cdot\left(1-p_{B L E R}\left(N_{P R B}, l_{M C S}\right)\right)
$$

where $r\left(N_{P R B}, l_{M C S}\right)$ is the maximum instantaneous data rate as a function of the number of Physical Resource Blocks $\left(N_{P R B}\right)$ and the modulation and coding scheme index $\left(l_{M C S}\right) . N_{P R B}$ has been set to 6 following the 3GPPP TR28.814 guidelines, and $l_{M C S}$ coincides with the 15 available Channel Quality Indicator (CQI) indexes in LTE. The indexes $l_{M C S}$ are set according to the distance to the BS; more robust MCS are needed as the distance increases. $r\left(N_{P R B}, \quad l_{M C S}\right)=T B S / T_{T B S}$ is calculated mapping the indexes $l_{M C S}$ to the Transport Block Size (TBS) using the table reported in 3GPP TR36.213. $T_{T B S}$ is the TBS duration which is equal to $0.5 \mathrm{~ms}$ in LTE. The block error rate experienced for $N_{P R B}$ and $l_{M C S}\left(p_{B L E R}\left(N_{P R B}, l_{M C S}\right)\right)$ has been set to $10 \%$, which coincides with the target BLER when reporting CQI values as defined in 3GPP TR36.213.

Following [15], the D2D IEEE 802.11g throughput can be computed as a function of the distance ( $d$, in meters) between SN and MR:

$$
T R_{n>n}(d)=\text { DataRate }(d) \cdot E f f \cdot(1-P E R(d))
$$

DataRate is one of the IEEE $802.11 \mathrm{~g}$ data rates: $\{54,48,36,24$, 18, 12, 9, 6; 11, 5.5, 2, 1\} Mbps). PER is the Packet Error Ratio. Both DataRate and PER models are empirically derived in [15]. Eff is the channel efficiency that represents the

\footnotetext{
${ }^{3}$ 3GPP (TR 22.803) considers 802.11 technologies as well as cellular technologies (e.g. LTE-Direct) for device-to-device communications.
} 
effective time that the 802.11g channel is used to transmit data and is modelled in [16].

The energy consumed by the store and carry process $\left(E_{S C}\right)$ has been modelled following [17]. This energy includes the energy consumption at DRAM and NAND flash memories. Mobile devices automatically store data packets received from the wireless interface in a storage unit (DRAM). The storage energy consumption can be reduced by transferring the information to the internal NAND flash unit. In this case, the information needs to be transferred back to the DRAM when the mobile device starts the cellular forwarding process. We assume that mobile devices always transfer the information to the internal NAND flash unit following the conclusions reached in [17]. In this context, $E_{S C}$ is computed considering the power state transitions of the DRAM and NAND flash units when reading and writing information. The $E_{S C}$ estimation also takes into account the DRAM and NAND power consumption in idle state when the information is stored. The energy consumption values for the two storage units at the different states have been obtained from [17].

\section{Influence of traffic characteristics on the energy benefits of opportunistic MCN communications}

The optimum configuration of opportunistic MCN communications and their energy consumption depend on the traffic characteristics $T$ and $F$. This study therefore investigates the impact of such characteristics on the total energy consumption, and how opportunistic MCN communications should be configured to exploit traffic characteristics in order to reduce the energy consumption. The study is conducted through numerical resolution of the analytical framework developed in the previous section. The optimization problem has been solved by means of brute force techniques implemented in Matlab, for all possible distances between SN and BS, and for a wide set of values of $T$ and $F$.

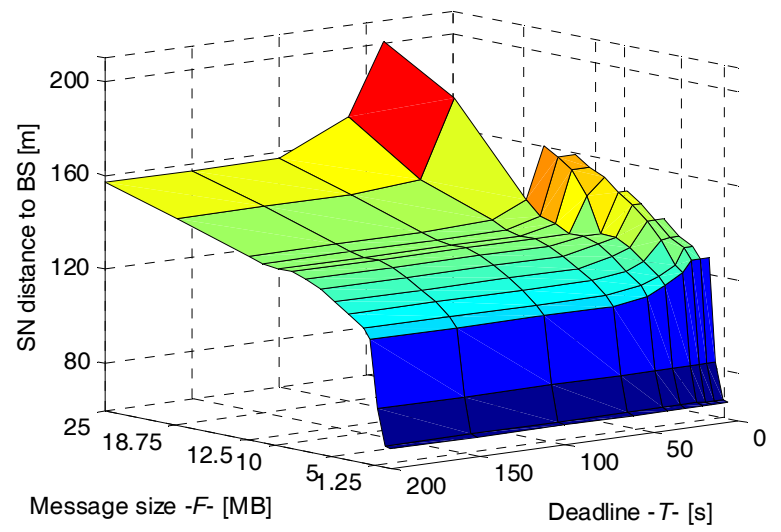

Figure $2 \mathrm{SN}$ distance to the BS from which 2-hop opportunistic MCN communications are more energy efficient than single-hop cellular communications. The distance is depicted as a function of the size of the message to be transmitted $(F)$ and the trafficdependent deadline $(T)$.
The energy consumed by opportunistic MCN communications is first compared against that consumed in traditional single-hop cellular ( $\mathrm{SH}$, direct cellular connection between $\mathrm{SN}$ and $\mathrm{BS})^{4}$. Figure 2 shows the $\mathrm{SN}$ distance to the BS from which 2-hop opportunistic MCN communications are more energy efficient than single hop (SH) cellular communications. The results are shown for a variety of $F$ and $T$, and have been obtained considering a cell radius of $1 \mathrm{~km}$ and mobile MRs moving towards the BS at pedestrian speed $(2 \mathrm{~m} / \mathrm{s})$. The depicted results show that the SN distance to the BS from which opportunistic MCN communications are more efficient than $\mathrm{SH}$ communications increases with increasing values of $F$. This means that with messages of larger size, opportunistic MCN communications can be more energy efficient if SN is not close to the BS. This is due to the fact that large messages increase the $E_{D 2 D}$ and $E_{S C}$ energy consumption levels in the opportunistic MCN connection. To reduce the overall energy consumption with respect to $\mathrm{SH}$, high energy savings from the MR-BS cellular transmission are hence necessary to compensate the increased $E_{D 2 D}$ and $E_{S C}$ levels. Opportunistic MCN communications can achieve such savings with increasing SN-BS distances because of the lower $\mathrm{SH}$ energy efficiency at large distances to the BS, and the use of store, carry and forward schemes with mobile relays. This is actually reflected in Figure 3 that shows the total energy consumption for $\mathrm{SH}$ and opportunistic MCN communications as a function of the SN-BS distance ( $F=5 \mathrm{MB}$ and $T=150 \mathrm{~s}$ ). Figure 3 shows that an optimum configuration of opportunistic MCN communications can result in important reductions in total energy consumption for SN-BS distances larger than $125 \mathrm{~m}$. For a SN distance to the BS equal to $200 \mathrm{~m}$, opportunistic MCN communications reduce by $60 \%$ the energy consumption compared with traditional SH communications. This gain increases above $90 \%$ for distances between SN and BS higher than 290m. Figure 3 has been obtained considering LTE for the cellular transmissions. Simulations were also conducted with HSPA, and the same trends were observed with slightly smaller energy gains for opportunistic MCN communications compared to when using LTE. This is the case because of the higher LTE transmission rates that benefit opportunistic MCN communications as the MR moves towards the BS and forwards the information to the cellular network.

\footnotetext{
${ }^{4}$ The energy consumption for $\mathrm{SH}$ communications is computed using the model for energy consumption of cellular transmissions reported in the previous section, and considering a direct cellular SN-BS connection (i.e. there is no D2D transmission and store and carry process).
} 


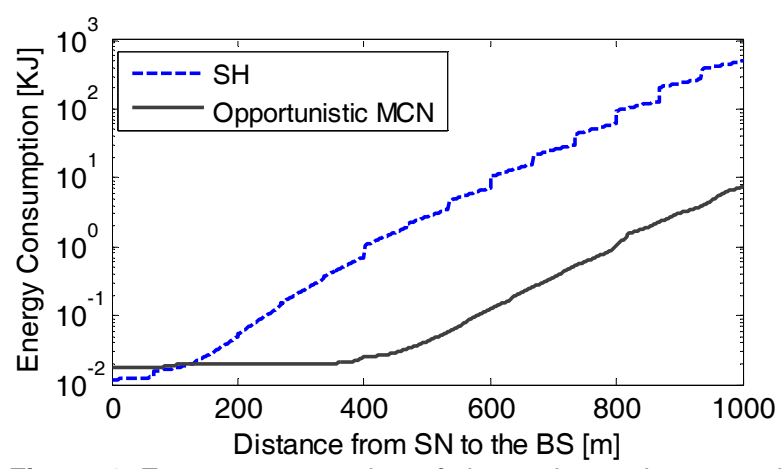

Figure 3 Energy consumption of the end-to-end opportunistic $M C N$ communications as a function of the distance from $\mathrm{SN}$ to the $B S$ when $F=5 M B$ and $T=150$ s.

Figure 2 also shows that the SN distance to the BS from which opportunistic MCN communications are more energy efficient than $\mathrm{SH}$ increases when the message deadline $T$ is small. This is the case because small deadlines do not provide sufficient time to exploit the store and carry process, and the MR forwards the message to the BS as soon as received. When $T$ increases, the SN-BS distance from which opportunistic MCN achieves higher energy efficiency than SH does not vary with $T$. However, $T$ has an impact on the average reduction in total energy consumption of opportunistic MCN compared to $\mathrm{SH}$. Such impact is shown in Table 1 as a function of the distance between SN and BS. As previously discussed, opportunistic MCN communications do not provide an energy gain when SN is close to the BS (SN-BS distances below 100 meters); Table 1 shows that this trend is independent of the deadline $T$. When the SN-BS distance increases, opportunistic MCN communications can significantly reduce the energy consumption compared to $\mathrm{SH}$, reaching reduction levels near $100 \%$ under certain conditions. In fact, the obtained results show that for a fixed deadline $T$, opportunistic MCN communications achieve its higher energy gains compared to $\mathrm{SH}$ when the distance from SN to the BS increases. When this distance increases, opportunistic MCN communications achieve their higher possible energy gains compared to $\mathrm{SH}$ when $T$ also increases, but up to a certain limit. For example, when $\mathrm{SN}$ is at a distance to the BS between 401 and $500 \mathrm{~m}$, the maximum energy reduction is reached for $T=200 \mathrm{~s}$, and no further gains are achieved for higher values of $T$. Increasing $T$ allows reducing the D2D distance at which SN should start the transmission to $\mathrm{MR}$, as well as the distance to the BS at which MR should start the cellular transmission to reduce the total energy consumption. Reducing such distances decreases the energy consumption in the D2D and cellular transmissions but increases the energy consumption of the store and carry process at the MR. The results depicted in Table 1 clearly show that for a given SN-BS distance there is a $T$ threshold from which the D2D and cellular transmission energy savings do not compensate the increased store and carry energy consumption levels. Table 1 does not show any values when $T=40 \mathrm{~s}$ and the
SN-BS distance increases beyond $700 \mathrm{~m}$. This is the case because under these conditions, it is not possible to establish an opportunistic MCN connection that can transmit the complete 10MB message before the $T$ deadline expires. Since such connection is not possible, a comparison with $\mathrm{SH}$ is not feasible.

\begin{tabular}{|c|c|c|c|c|}
\hline \multirow{2}{*}{ SN-BS distance [m] } & \multicolumn{4}{|c|}{$\boldsymbol{T}[\mathbf{s}]$} \\
\cline { 2 - 5 } & $\mathbf{4 0}$ & $\mathbf{1 0 0}$ & $\mathbf{2 0 0}$ & $\mathbf{3 0 0}$ \\
\hline $1-100$ & -34.1 & -34.1 & -34.1 & -34.1 \\
\hline $101-200$ & 4.7 & 11.5 & 11.5 & 11.5 \\
\hline $201-300$ & 55.3 & 69.1 & 70.1 & 70.1 \\
\hline $301-400$ & 72.7 & 88.0 & 91.3 & 91.3 \\
\hline $401-500$ & 81.3 & 94.3 & 97.8 & 97.8 \\
\hline $501-600$ & 80.6 & 94.5 & 98.9 & 99.2 \\
\hline $601-700$ & 82.7 & 95.4 & 99.4 & 99.8 \\
\hline $701-800$ & - & 95.5 & 99.3 & 99.9 \\
\hline $801-900$ & - & 95.7 & 99.5 & 99.9 \\
\hline $901-1000$ & - & 94.8 & 99.4 & 99.9 \\
\hline
\end{tabular}

Table 1 Average energy reduction (in \%) of opportunistic MCN communications compared to single-hop cellular communications for values of $T$ and $F=10 \mathrm{MB}$.

The energy gains of opportunistic MCN communications depend on an adequate selection of the MR. For example, selecting an MR that provides the highest progress towards the BS can reduce the energy consumed in the cellular transmission, but this is done at the expense of higher energy consumption levels in the D2D transmission that can increase the total energy consumption. It is therefore necessary to derive the optimum MR location that minimizes the overall energy consumption, and analyse its dependence with the traffic service characteristics. Figure 4 shows the optimum MR location to start the D2D transmission as a function of the distance between SN and BS. The MR location is represented by means of the distance between $\mathrm{SN}$ and MR. Figure 4.a shows for example that if SN is located $600 \mathrm{~m}$ away from the BS, the MR should be ideally located $88 \mathrm{~m}$ away from $\mathrm{SN}$ in the direction of the BS in order to minimize the total energy consumption. The results depicted in Figure 4.a show that the distance between SN and MR that minimizes the energy consumption increases with the distance between SN and BS, and that it does not vary with the message size $F$ (fixing the message deadline $T$ to 100s). On the other hand, Figure 4.b shows that, for a given location of SN, the distance from $\mathrm{SN}$ to MR that minimizes the overall energy consumption decreases as $T$ increases ( $F$ is set to $6.25 \mathrm{MB}$ ). With increasing values of $T$, MR has more time to store and carry the information, and therefore can reduce the $\mathrm{D} 2 \mathrm{D}$ distance with $\mathrm{SN}$, and hence the total energy consumption. On the other hand, when the traffic service is characterized by lower $T$ deadlines, opportunistic MCN communications should increase the D2D distance to start the cellular forwarding process at closer distances to the $\mathrm{BS}$ and reduce the total energy consumption. 

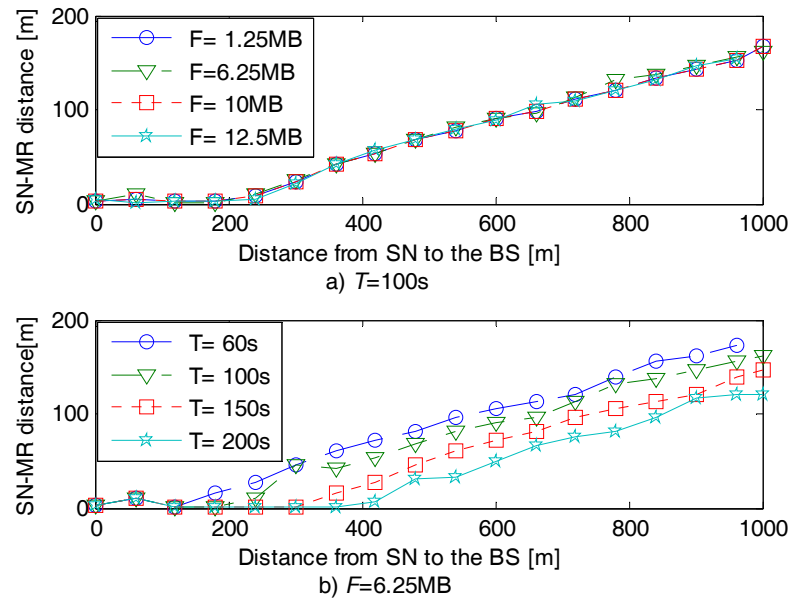

Figure 4 Optimum MR location to minimize the total energy consumption for opportunistic MCN communications. The MR location is shown as a function of the distance from SN to the BS and varying service characteristics ( $F$ and $T$ ).

\section{Mobile video traffic}

The relevance of mobile video traffic in current and forecasted cellular data traffic statistics requires a specific analysis of the energy benefits that could be achieved when using opportunistic MCN communications to transmit mobile video traffic. In fact, opportunistic MCN communications can take advantage of the buffers that video players implement to keep the video content ahead of playback time. This allows grouping the video content fragments to postpone their transmission at no cost to the perceivable QoE as long as the playback is not interrupted. Figure 5 compares the average energy consumption in the transmission of a mobile video file using traditional SH cellular communications and opportunistic MCN communications. The results are shown for the case in which a traditional adaptive streaming (AS) scheme is used to upload the multiple video fragments [8] (bars marked as AS). The opportunistic MCN energy consumption is also evaluated when video content fragments are grouped into messages of $1 \mathrm{MB}, 2 \mathrm{MB}, 3 \mathrm{MB}$ and $4 \mathrm{MB}$. In this case, the video messages are equivalent to $10 \mathrm{~s}, 20 \mathrm{~s}, 30 \mathrm{~s}$ and $40 \mathrm{~s}$ of playback [8] respectively. As a result, a $1 \mathrm{MB}-10 \mathrm{~s}$ traffic configuration requires that the opportunistic MCN transmission of the $1 \mathrm{MB}$ message from SN to BS concludes before 10s to avoid the video playback to freeze. This study considers the average YouTube video size (7.6MB) reported in [18]. In the case of the $1 \mathrm{MB}-10 \mathrm{~s}$ traffic configuration, eight opportunistic MCN transmissions from $\mathrm{SN}$ to $\mathrm{BS}$ are necessary to complete the video transmission (seven transmissions of $1 \mathrm{MB}$ plus one transmission of $0.6 \mathrm{MB}^{5}$ ). A $4 \mathrm{MB}-40 \mathrm{~s}$ traffic configuration

\footnotetext{
${ }^{5}$ We consider that the time available to transmit the $0.6 \mathrm{MB}$ video fragment from SN to BS is also equal to 10s. This is the case because the time available to transmit this fragment is related to the buffered
}

would only require two transmissions $(1 \mathrm{x} 4 \mathrm{MB}+1 \mathrm{x} 3.6 \mathrm{MB})$ with the maximum time available (deadline $T$ ) for each one equal to 40s. Each of these opportunistic MCN transmissions is initiated at the same location of the SN but uses different MRs (always at the same location for a given traffic configuration, Figure 4.a) as MRs are mobile. The energy consumption levels shown in Figure 5 for opportunistic MCN communications correspond then to the sum of the energy consumption in each one of the transmissions necessary to complete the video file transference. The energy consumption values shown in Figure 5 have been averaged for all possible distances between SN and BS considering a cell radius of $1 \mathrm{~km}$ and MRs moving towards the BS at $2 \mathrm{~m} / \mathrm{s}$.

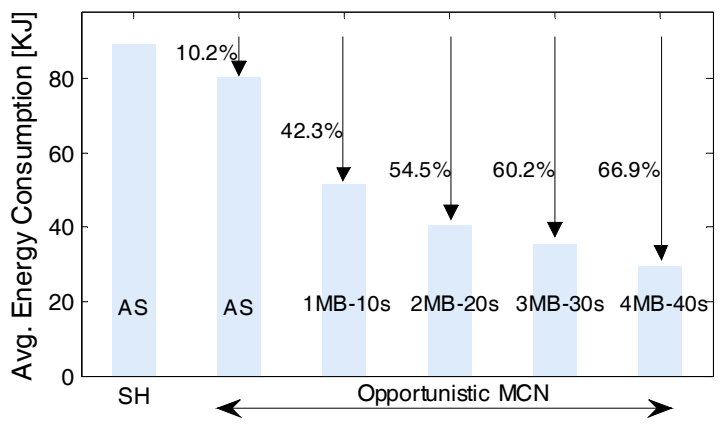

Figure 5 Average energy consumption to transmit a $7.6 \mathrm{MB}$ video file. Video content fragments of $1 M B, 2 M B, 3 M B$ and $4 M B$ (equivalent to 10s, 20s, 30s and 40s of playback) are grouped in the case of opportunistic MCN. The figure shows the reduction (in \%) in average energy consumption that opportunistic MCN communications can achieve compared to traditional $\mathrm{SH}$ communications for each video traffic configuration.

Figure 5 shows that opportunistic MCN communications can considerably reduce the average energy consumption with respect to $\mathrm{SH}$. The reduction is particularly relevant when video content fragments are grouped. In fact, the reduction increases with the number of video content fragments being grouped. For example, the reduction increases from $42.26 \%$ with the $1 \mathrm{MB}-10$ s traffic configuration to $66.87 \%$ with the $4 \mathrm{MB}-40$ s traffic configuration. This is due to the fact that opportunistic MCN communications can benefit from increasing values of the transmission deadline $T$ since the MR would have more time to store and carry the information, and therefore reduce the D2D distance, the cellular distance with the BS, and finally the total energy consumption. The message deadline $T$ in mobile video traffic, and therefore the energy benefits of opportunistic MCN communications, increase with the amount of video content fragments that are grouped. It should be noted that this is at the expense of a higher delay in the transmission of the first message, which can determine the start of the playback. For example, the first message (set of grouped video content fragments) is uploaded to the BS before

data in the playback from the previous transmission (1MB video fragment with 10 s of playback). 
10s elapse in the case of the 1MB-10s traffic configuration. On the other hand, it could take up to 40 s to receive the first $4 \mathrm{MB}$ message in case of the 4MB-40s traffic configuration.

\section{Conclusions}

Mobile data traffic is continuously growing with mobile users increasingly taking a prosumer role. Cellular networks have to be prepared to efficiently accommodate the expected growth levels. Some of the services driving the mobile data growth are characterized by certain delay tolerance. The authors propose to exploit such delay tolerance to improve the energy-efficiency of cellular networks through the use of opportunistic networking policies. In this context, this study has analyzed the impact of traffic characteristics of delay tolerant services on the energy gains that can be reached with opportunistic MCN communications compared to traditional $\mathrm{SH}$ cellular communications. The study has also investigated how opportunistic MCN communications should be configured to exploit the traffic delay tolerance in order to reduce the total transmission energy consumption. The obtained results have shown that opportunistic MCN communications can significantly reduce the energy consumption compared to traditional single-hop cellular communications for delay tolerant services. The reduction can even be higher than $90 \%$ under certain conditions. In particular, the reduction levels increase with increased traffic delay tolerance thresholds and the increased distance of the source node to the serving base station. Particular attention has been paid to the case of mobile video traffic that accounts for a very significant portion of current and forecasted mobile data traffic. The conducted analysis has shown that by grouping video content fragments, mobile video traffic and opportunistic MCN communications can provide significant energy savings in cellular networks at no cost to the perceived QoE.

\section{References}

[1] R. Baldemair, et al, "Evolving Wireless Communications: Addressing the Challenges and Expectations of the Future," IEEE Vehicular Technology Magazine, vol. 8, no. 1, Mar. 2013.

[2] M.J. Yang, S.Y. Lim, H.J. Park, and N.H. Park, "Solving the data overload: Device-to-device bearer control architecture for cellular data offloading," IEEE Vehicular Technology Magazine, vol.8, no.1, pp.31-39, Mar. 2013.

[3] H. Nishiyama, M. Ito, and N. Kato, "Relay-by-smartphone: realizing multihop device-to-device communications," IEEE Communications Magazine, vol. 52, no. 4, pp. 56-65, Apr. 2014.

[4] J. Gozalvez, and B. Coll-Perales, "Experimental evaluation of multihop cellular networks using mobile relays," IEEE Communications Magazine, vol.51, no.7, pp.122-129, Jul. 2013.

[5] L. Pelusi, A. Passarella, and M. Conti, "Opportunistic networking: data forwarding in disconnected mobile ad hoc networks," IEEE Communications Magazine, vol.44, pp.134-141, Nov. 2006.

[6] B. Coll-Perales, J. Gozalvez, and V. Friderikos, "Opportunistic Networking for Improving the Energy Efficiency of Multi-Hop Cellular Networks," Proceedings of the IEEE CCNC, pp.10801085, Las Vegas, USA, 10-13 Jan. 2014.
[7] B. Coll-Perales, J. Gozalvez and V. Friderikos, "Energy-efficient opportunistic forwarding in multi-hop cellular networks using device-to-device communications", Transactions on Emerging Telecommunications Technologies, early view, Aug. 2014.

[8] P. Ameigeiras, J.J. Ramos-Muñoz, J. Navarro-Ortiz, and J.M. López-Soler, "Analysis and modelling of YouTube traffic," Transactions on Emerging Telecommunications Technologies, vol.23, no.4, pp.360-377, Jun. 2012.

[9] P. Kolios, V. Friderikos, and K. Papadaki, "Energy-Aware Mobile Video Transmission Utilizing Mobility," IEEE Network, vol.27, no.2, pp.34-40, Mar. 2013.

[10] J.I. Moreno-Novella, and F.J. Gonzalez-Castaño, "Resource Management in Satellite Networks (Chap. 3): QoS Requirements for Multimedia Services,” Springer, pp.67-94, 2007.

[11] B. Bing, "A fast and secure framework for over-the-air wireless software download using reconfigurable mobile devices," IEEE Communications Magazine, vol.44, no.6, pp.58-63, Jun. 2006.

[12] C. Troncoso, et al, "PriPAYD: Privacy-Friendly Pay-As-YouDrive Insurance," IEEE Transactions on Dependable and Secure Computing, vol.8, no.5, pp.742-755, Sept.-Oct. 2011.

[13] WINNER consortium, "D1.1.2 V.1.1. WINNER II channel models", WINNER European Research project Public Deliverable, Nov. 2007.

[14] P. Bertrand, J. Jiang, and A. Ekpenyong, "Link Adaptation Control in LTE Uplink," Proceedings of the IEEE VTC Fall, pp. 1-5, Quebec, Canada, 3-6 Sept. 2012.

[15] B. Coll-Perales, J. Gozalvez, and J. Sanchez-Soriano, "Empirical Performance Models for P2P and Two Hops Multi-hop Cellular Networks with Mobile Relays," Proceedings of the ACM PM2HW2N, pp.21-28, Barcelona, Spain, 3-8 Nov. 2013.

[16] A. Duda, "Understanding the Performance of 802.11 Networks", Proceedings of the IEEE PIMRC, pp.1-6, Cannes, France, 15-18 Sept. 2008.

[17] B. Zhao, and V. Friderikos, "Optimal stopping for energy efficiency with delay constraints in Cognitive Radio networks," Proceedings of the IEEE PIMRC, pp.820-825, Sydney, Australia, 9-12 Sept. 2012.

[18] X. Cheng, J. Liu, and C. Dale, "Understanding the Characteristics of Internet Short Video Sharing: A YouTube-Based Measurement Study," IEEE Transactions on Multimedia, vol.1, no.5, pp.11841194, Aug. 2013.

\section{Acknowledgements}

This work is supported in part by the Spanish Ministry of Economy and Competitiveness and FEDER funds (TEC201126109), and the Local Government of Valencia with reference ACIF/2010/161 and BEFPI/2012/065. This work is also partially supported by the European Commission in the framework of the FP7 Mobile Opportunistic Traffic Offloading (MOTO) under grant agreement number 317959.

Baldomero Coll-Perales (bcoll@umh.es) received a telecommunications engineering degree from the Miguel Hernández University of Elche (UMH) in 2008. He received the best student awards in telecommunications engineering by both the UMH and the professional organization of telecommunications engineers. He then joined the UWICORE research laboratory to work on the development of networking and communication protocols for mobile relaying multi-hop cellular networks under the Opportunities, m-HOP and ICARUS research projects. He is currently pursuing his Ph.D. studies with a fellowship from the Valencia regional 
THIS IS AN AUTHOR-CREATED POSTPRINT VERSION.

The final publication is available at http://ieeexplore.ieee.org/xpl/articleDetails.jsp?arnumber=7108152

DOI 10.1109/MVT.2015.2411414

government. His research focuses on multi-hop cellular networks, including networking, connectivity and resource management aspects.

Javier Gozalvez (j.gozalvez@umh.es) received an electronics engineering degree from the Engineering School ENSEIRB (Bordeaux, France), and a PhD in mobile communications from the University of Strathclyde, Glasgow, U.K. Since October 2002, he is with the Miguel Hernández University of Elche, Spain, where he is currently an Associate Professor and Director of the UWICORE laboratory. At UWICORE, he leads research activities in the areas of multi-hop cellular networks, vehicular networks, resource management and heterogeneous networks, and wireless industrial communications. He has published over 110 papers in international conferences and journals. He is an elected member to the Board of Governors (2011-2017) and Executive Vice President of the IEEE Vehicular Technology Society. He is an IEEE Distinguished Lecturer for the IEEE Vehicular Technology Society. He currently serves as Mobile Radio Senior Editor of IEEE Vehicular Technology Magazine, and previously served as AE of IEEE Communication Letters. He is the General Co-Chair for the IEEE VTC-Spring 2015 conference in Glasgow (UK), and was General Co-Chair of the ACM VANET 2013, ACM VANET 2012 and 3rd ISWCS 2006. He also was TPC CoChair for 2011 IEEE VTC-Fall and 2009 IEEE VTC-Spring. He is also the founder and General Co-Chair of the IEEE International Symposium on Wireless Vehicular communications (WiVeC) in its 2007, 2008, and 2010 editions.

Oscar Lazaro (olazaro@innovalia.org) is Managing Director of Innovalia Association. He has led Innovalia, since 2000, in many large European research initiatives. He holds several patents, international research awards and large list of international publications in major conferences and journals. Dr Oscar Lazaro has more than 15 years of experience in the ICT field and is leading research and product development activities of Innovalia for Smart Cities and Factories of the Future domains. Oscar Lazaro is also Visiting Professor at the Electrical and Electronic Engineering Department of the University of Strathclyde in the area of wireless \& mobile communications. He is since 2013 chairman of the European Digital Business Innovation (DBI) Community and a permanent representative of Innovalia in EFFRA and Future Internet (FI-PPP) Steering Boards. His fields of expertise cover wireless communications, security, Future Internet and sensing enterprise technologies with a focus on applications in smart cities, manufacturing and enterprise domains.

Miguel Sepulcre (msepulcre@umh.es) received a telecommunications engineering degree in 2004 and a Ph.D. in communications technologies in 2010, both from the Miguel
Hernández University of Elche (UMH), Spain. In 2004, he spent six months at the European Space Agency (ESA) in Noordwijk (The Netherlands) working on the communications physical layer of earth exploration satellites. In 2006, he obtained a Ph.D. fellowship from the Valencian regional government and joined the UWICORE research laboratory to work on vehicular communications. He was awarded by the COIT (Spanish official association of Telecommunication Engineers) with the ONO prize to the best Ph.D. thesis. He is currently a research fellow at the UWICORE Laboratory of $U M H$, working on vehicular communications, radio resource management, modelling and simulation of wireless communications systems. 